\title{
STUDENT PREDICTION SYSTEM FOR PLACEMENT TRAINING USING FUZZY INFERENCE SYSTEM
}

\author{
Ravi Kumar Rathore ${ }^{\mathbf{1}}$ and J. Jayanthi ${ }^{\mathbf{2}}$ \\ School of Computer Science and Engineering, Lovely Professional University, India
}

\begin{abstract}
Proposed student prediction system is most vital approach which may be used to differentiate the student data/information on the basis of the student performance. Managing placement and training records in any larger organization is quite difficult as the student number are high; in such condition differentiation and classification on different categories becomes tedious. Proposed fuzzy inference system will classify the student data with ease and will be helpful to many educational organizations. There are lots of classification algorithms and statistical base technique which may be taken as good assets for classify the student data set in the education field. In this paper, Fuzzy Inference system has been applied to predict student performance which will help to identify performance of the students and also provides an opportunity to improve to performance. For instance, here we will classify the student's data set for placement and non-placement classes.
\end{abstract}

Keywords:

Classification, Fuzzy Inference System, MATLAB

\section{INTRODUCTION}

Student prediction system is most important approach as it classifies large set of student data is very difficult so many organizations suffer from this problem and this would be serious concern. If we are going to classify large set of data set of student on excel sheet it will take lot of time and if we use any programming language, it is also very difficult to code for many conditions and classify big data set. This is the serious issue which frequently occurs during classification of student big data set or predicting them for desire purpose. Fuzzy expert system may provide very easy way to classify large set of student data set or we can predict large set of students as well as individual student also. Fuzzy provide a good platform for making conditions or rules and we can easily implement this technique by using fuzzy inference system.

\section{LITERATURE SURVEY}

Many systems and numerous algorithm exists which produce to constructs classification model for student prediction such as Hidayah et.al [1] where researcher used ANFIS techniques with Fuzzy logic toolbox for classify the students on the basis of their academic performance. They attempted and mapped the conditions according to created rules for removing difficulty for classifies the students. Devi et al. [2] used Neuro-Fuzzy inference system with hybrid learning algorithm to advance the prophecy based on the conservative replica using questioner. They created some rules on the basis of their parameters. Malaise [3] used multi agent data mining technique for predicting student performance. In that paper, researcher induced performance of the students based on their performance and predicts accuracy as well by using multiple techniques such as Adaboost, C4.5 algorithm. Qiu and Sapiro [4] done learning transformation for clustering and classification. They worked on low-rank transformation for sub space clustering and classification. Researcher transformed highdimensional data space into low dimensional subspace like Sharma [5] proposed a literature survey using data mining. Data mining technique has been used by many researchers for extracting information within the large set of data. Barrak and Razgan [6] used data mining technique to predict students final GPA in educational based on their grades in previous courses. They collected the students' performance data based on their past performance. In that data their CGPA and their grades are mentioned of all courses they collected data of female students in that total students were 236 and then applied tree algorithm for inventing the classification rules. Padav and Pal [7] collected data from VBS purvanchal university Uttar Pradesh and they collected the data of 90 students which were from MCA department and researcher apply some algorithm on that data for classification. Kashy et al. [8] predict the student performance using of data mining methods with the educational web based system on the basis of student CGPA. Khan et al. [9] predicts the final grade of the secondary school students which has proven effective classification system. Researcher classifies the student's marks and grades in different subjects using data mining techniques. These data mining technique are classification, prediction, Decision Tree and J48. Talwar and Kumar [10] discussed machine learning methodology in artificial intelligence. Researcher also discussed about learning concept like pattern recognition and different machine learning algorithms such as naive basis classifier, trees, SVM, nearest method, discriminant analysis and more. Researcher consults the concepts of these machine learning algorithm that these algorithm how much efficient for classification as well as evaluation also.

\section{FUZZY INFERENCE SYSTEM}

\subsection{FUZZY SYSTEM}

Fuzzy logic is basically a logic which provides the simple recognition of truth and false values these values represent in propositions same here with the fuzzy logic, propositions can be represented with truthfulness and falsehood values. Just simple take an example of truth and false such as there is a statement, today is sunny, it might be $100 \%$ true if there are no clouds so we cannot say it is $100 \%$ correct but it may be $80 \%$ correct or true if it is hazy and if it rains all day so it is clear that it is $0 \%$ true. The idea of fuzzy logic is an approach to computing degree of truth rather than true or false ( 1 or 0 ) Boolean logic. Fuzzy logic is an extension of multi valued logic of propositional values into a set of membership degree it is known as membership function which 
maps the predicates into fuzzy sets or ordered set of fuzzy pairs called fuzzy relation. Let us take an example of fuzzy logic of slowest, slow, fast, fastest. In an every problem in fuzzy logic must represent the fuzzy sets. So we will define the range for these possibilities range should be like this for Slowest - [0.0 - 0.25], Slow - [0.25 - 0.50], Fast - [0.50-0.75] and Fastest - [0.751.00].

Fuzzy Logic is a logic which is used in data mining it gives approximate result rather than exact result. Basically fuzzy is an extension of crisp set there were some limitation in crisp for removing them one new method were invented which was fuzzy. Fuzzy is not work on probability it is work on uncertainty. Fuzzy logic is a way to represent linguistic variable and subjective attributes or variables of the real world in computing. Basically fuzzy provides uncertainty behavior not probability.

Uncertainty in fuzzy logic it represents by two ways first one is stochastic uncertainty and lexical uncertainty. In the fuzzy logic we use linguistic variable. A linguistic variable associate with word and it can be any sentences by using belief functions which is known by membership function. For making a fuzzy system use fuzzy inference system and create some rules on the basis of given parameters rules should be like this If and Then, shown in Fig.1

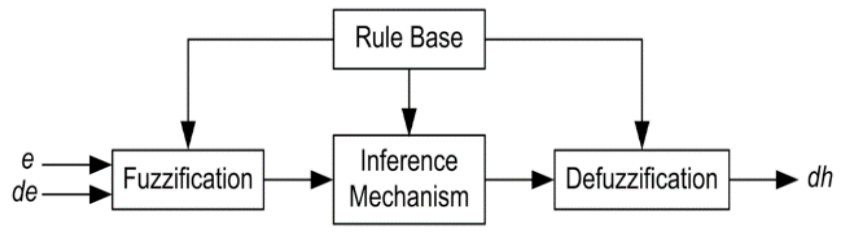

Fig.1. Fuzzy logic basic diagram

\subsection{FUZZY MEMBERSHIP FUNCTION}

Membership function in fuzzy logic play important role because fuzzy set is a simplification of the pointer function in classical sets. It represents the degree of truth values. Often degree of truth can have mystified with probabilities. Membership function in fuzzy logic play important role because fuzzy set is a simplification of the pointer function in classical sets. It represents the degree of truth values. Often degree of truth can have mystified with probabilities. Membership function can be representing by many types such as Triangular Membership Function, Trapezoidal Membership Function, Gaussian Membership Function and Generalized Bell Membership Function, shown in Fig.2.

\subsection{FUZZY RULE}

Rules in fuzzy logic represent like a condition on the basis of rule it will give result whether result can be true or false. Rules write in the form of condition such as IF THEN, shown in Fig.3.

\subsection{FUZZY INFERENCE SYSTEM}

Fuzzy Inference System or FIS is a method of mapping some inputs space to an output space using Fuzzy Logic. It provides the very simple platform which can use easily. Fuzzy inference system provide making inputs and membership function and corresponding we can get output, shown in Fig.4.
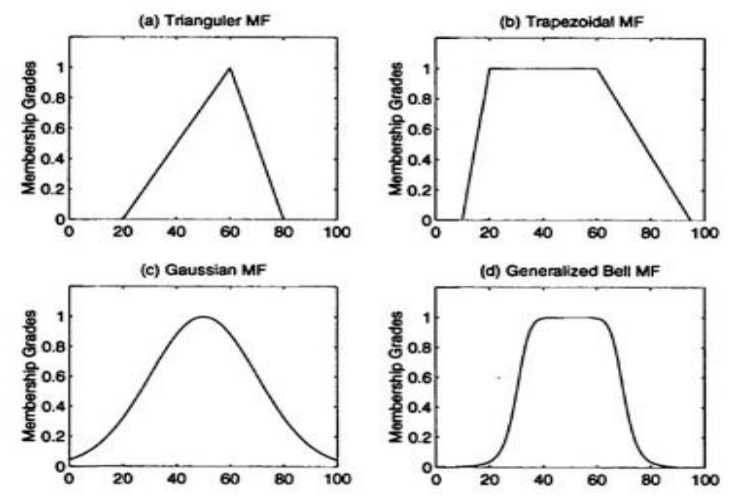

Fig.2. Fuzzy Membership Function
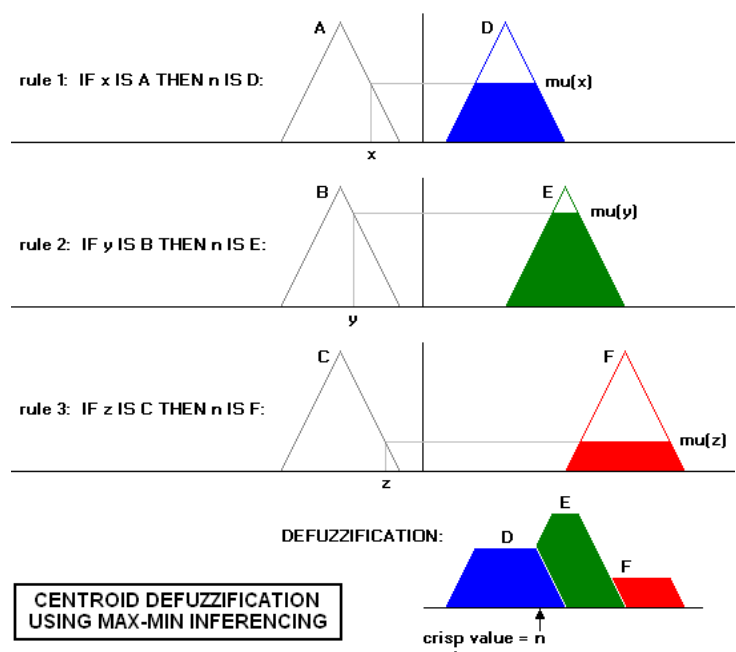

Fig.3. Rules representation in fuzzy logic

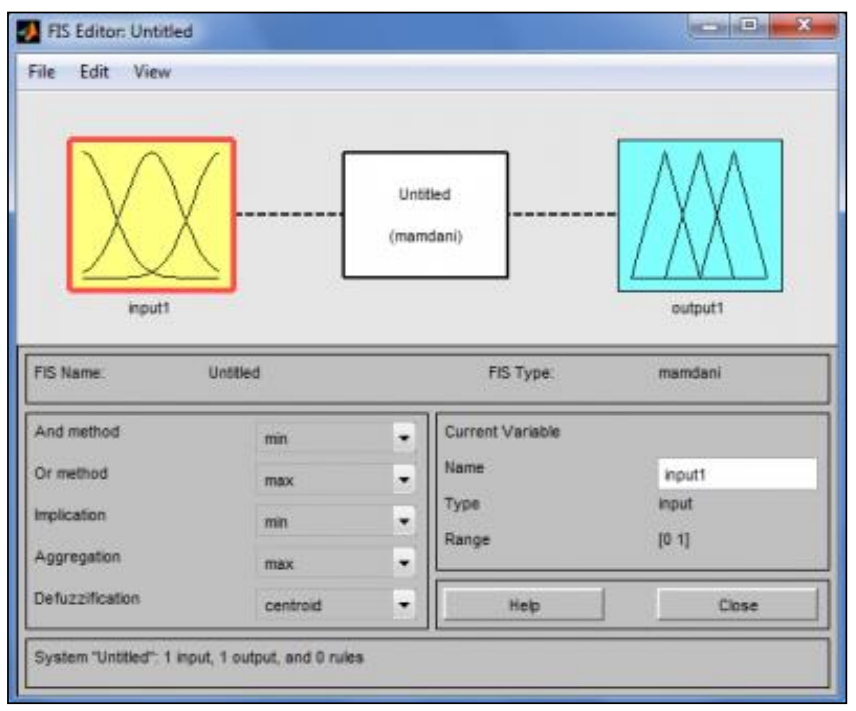

Fig.4. Fuzzy Inference system

\subsection{DEFUZZIFICATION}

During the defuzzification membership function defined over input variable which applied on their actual values that's why the degree of each rule argument determined 


\subsection{MATLAB TOOLBOX}

MATLAB is basically a tool which is very easy to use and it is a computer program that combines computations visualization power that makes very easier to use for engineers. MATLAB is a managerial program and in the MATLAB tool box we can also write scripts and commands also like other programming languages. MATLAB tool provides some most important predefined functions and commands which is very useful in perspective of user point of view. MATLAB environment provide some techniques such as managing variables, import and export data, perform calculations, generate plots, generate surface and we can develop and manage files also, shown in Fig.5.

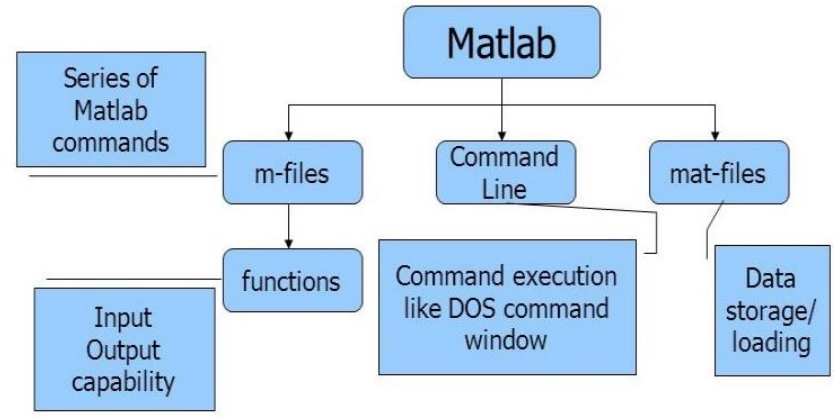

Fig.5. Basic function diagram of MATLAB

The main objective of this project is to classify large set of student data set using fuzzy logic and predicting student for placement training whether the student is eligible or not for placement training. As we discussed above we are taking two classes for final year student, classes are placement training and non-placement training. For the placement training those students will eligible those will get good marks or good CGPA in exam and remaining student will go for extra classes to improve their performance. From this approach we can predict all students in few times, if we want to classify all students on excel sheet so it will take lot of time, so these approaches are much better compare to other and it will help you.

This proposal may also able to judge the performance of the student continuously. The main advantage of this system is to improve accuracy and speed of the student by conducting an exam and decide some criteria to pass that exam if student fails in exam than some important classes or training will provide to the student it will help to student to improve itself. The advantage of the system is the accuracy of the prediction and speed of the result provided. So the fuzzy system provides the easy way to classify several numbers of student data set. By making a fuzzy inference system and create some rules which will predict the student for pre-defined classes such as placement training and non-placement training, shown in Fig.6.

For this system we collected 31 sample data set of M.Tech computer science student from our organization. In this data set put student registration number, name, 10th marks, 12th marks, B.Tech cgpa, M.Tech cgpa and number of backlogs. For classifying data set we skip some part of data set like registration number as well as name also here we take only academic marks and current backlog only and created more than 500 rules for predicting student performance for placement training on the basis of certain parameters. For example, if $\left(10^{\text {th }}\right.$ marks is poor $)$ and (12 $2^{\text {th }}$ marks is poor) and (B.Tech cgpa is poor) and (M.Tech cgpa is poor) and (backlog is less than 2) then (output is NO). From this fuzzy system can classify thousands number of student's dataset.

The main step of fuzzy based student prediction system is creating membership function for each input $\left(10^{\text {th }}\right.$ marks, $12^{\text {th }}$ marks, B.Tech CGPA, M.Tech CGPA, and number of backlogs) and creates output in terms of eligibility corresponding inputs. This fuzzy inference system is implemented in MATLAB tool box which is open source tool. And generated GUI interface which is like a page on which can calculate individual student result and predict his/her for placement training. The Fig.7 of membership function of each input given under as follows

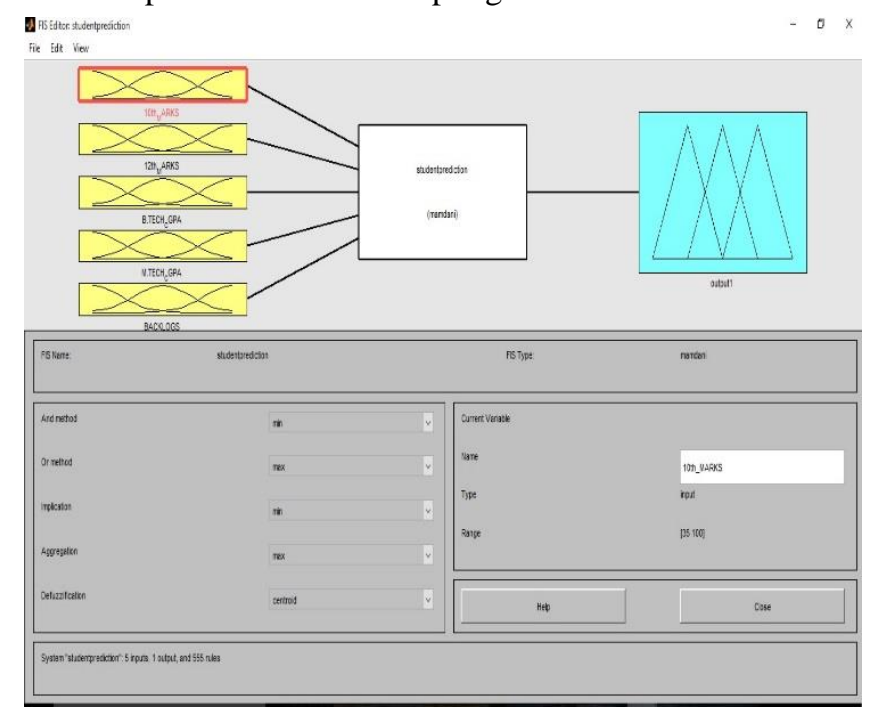

Fig.6. Fuzzy Inference System of each Input.

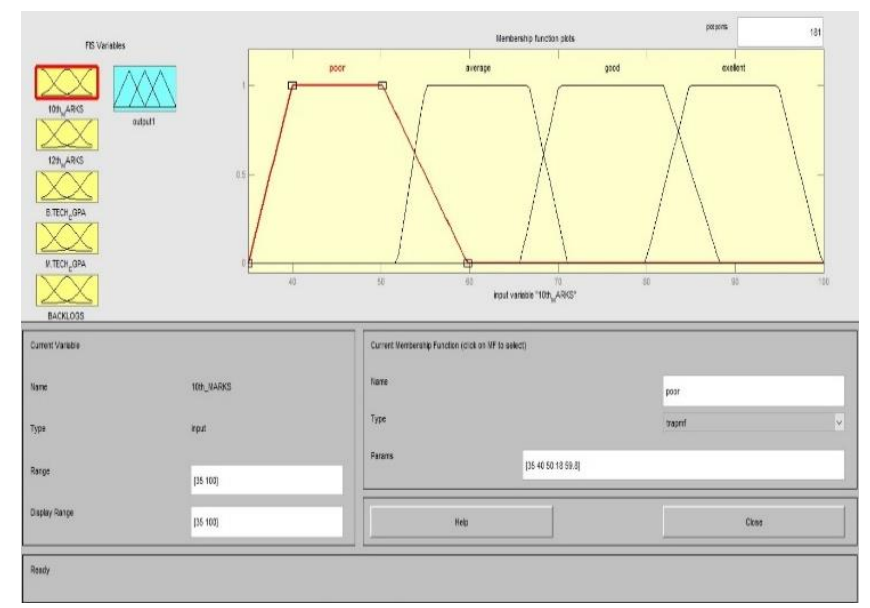

Fig.7. Membership function $-\left(10^{\text {th }}\right.$ marks $)$

The range of each membership functions for each input is defining by given Table.1.

Table.1. Input and Output Member Functions Range

\begin{tabular}{|c|c|}
\hline Inputs & $\begin{array}{c}\text { Number of Membership } \\
\text { Function }\end{array}$ \\
\hline $10^{\text {th }}$ Marks & 4 \\
\hline $12^{\text {th }}$ Marks & 4 \\
\hline
\end{tabular}




\begin{tabular}{|c|c|c|c|c|c|c|c|c|}
\hline \multicolumn{4}{|c|}{ B.Tech CGPA } & \multicolumn{5}{|c|}{4} \\
\hline \multicolumn{4}{|c|}{ M.Tech CGPA } & \multicolumn{5}{|c|}{4} \\
\hline \multicolumn{4}{|c|}{ Number of Backlogs } & \multicolumn{4}{|c|}{2} & \\
\hline \multicolumn{4}{|c|}{$\begin{array}{c}10^{\text {th }} \text { Input MF \& } \\
12^{\text {th }} \text { Input MF }\end{array}$} & \multicolumn{4}{|c|}{ Range } & \\
\hline \multicolumn{4}{|c|}{ Poor } & \multicolumn{5}{|c|}{$[35,40,50.18,59.8]$} \\
\hline \multicolumn{4}{|c|}{ Average } & \multicolumn{5}{|c|}{$[51,55.4,66.7,77.1]$} \\
\hline \multicolumn{4}{|c|}{ Good } & \multicolumn{5}{|c|}{$[65.7,70,81.4,88.3]$} \\
\hline \multicolumn{4}{|c|}{ Very Good } & \multicolumn{5}{|c|}{$[79.86,84.86,94.86,99.86]$} \\
\hline \multicolumn{4}{|c|}{$\begin{array}{l}\text { B.Tech Input MF \& } \\
\text { M.Tech Input MF }\end{array}$} & \multicolumn{5}{|c|}{ Range } \\
\hline \multicolumn{4}{|c|}{ Poor } & \multicolumn{5}{|c|}{$[4,4.5,5,5.5]$} \\
\hline \multicolumn{4}{|c|}{ Average } & \multicolumn{5}{|c|}{$[5.5,5.6,5.77,6.7]$} \\
\hline \multicolumn{4}{|c|}{ Good } & \multicolumn{5}{|c|}{$[6.564,6.8,7.41,7.73]$} \\
\hline \multicolumn{4}{|c|}{ Very Good } & \multicolumn{5}{|c|}{$[7,7.5,8,10]$} \\
\hline \multicolumn{4}{|c|}{ Backlog MF } & \multicolumn{5}{|c|}{ Range } \\
\hline \multicolumn{4}{|c|}{ Less than 2} & \multicolumn{5}{|c|}{$[-1,0,1,2.985]$} \\
\hline \multicolumn{4}{|c|}{ More than 2} & \multicolumn{5}{|c|}{$[2.15,2.849,6,6]$} \\
\hline \multicolumn{4}{|c|}{ Output MF } & \multicolumn{5}{|c|}{ Range } \\
\hline \multicolumn{4}{|c|}{ Eligible } & & {$[0$,} & $2.1,4,4]$ & & \\
\hline & Not-El & gible & & {$[0.0$} & $318,0.0$ & $318,0.0$ & 0318,2 & \\
\hline 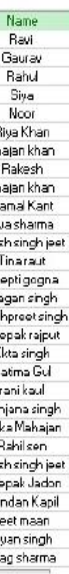 & 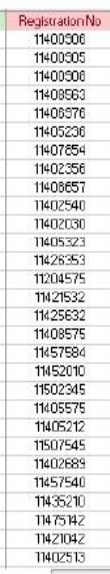 & 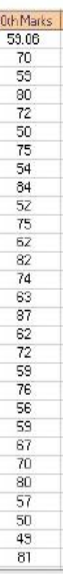 & 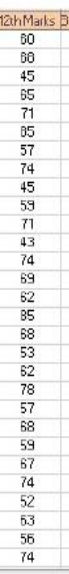 & 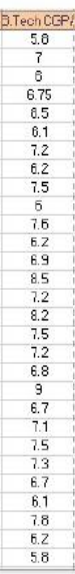 & 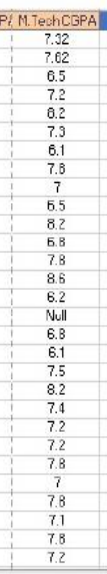 & 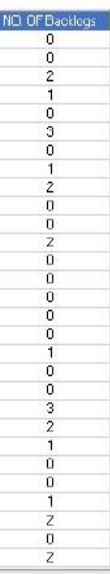 & 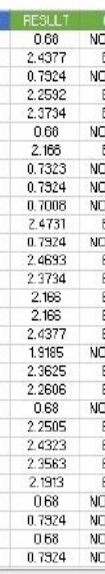 & 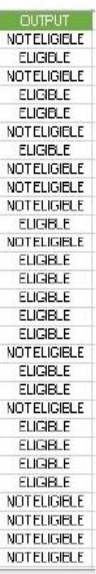 \\
\hline
\end{tabular}

Fig.8. Output of classified student dataset results

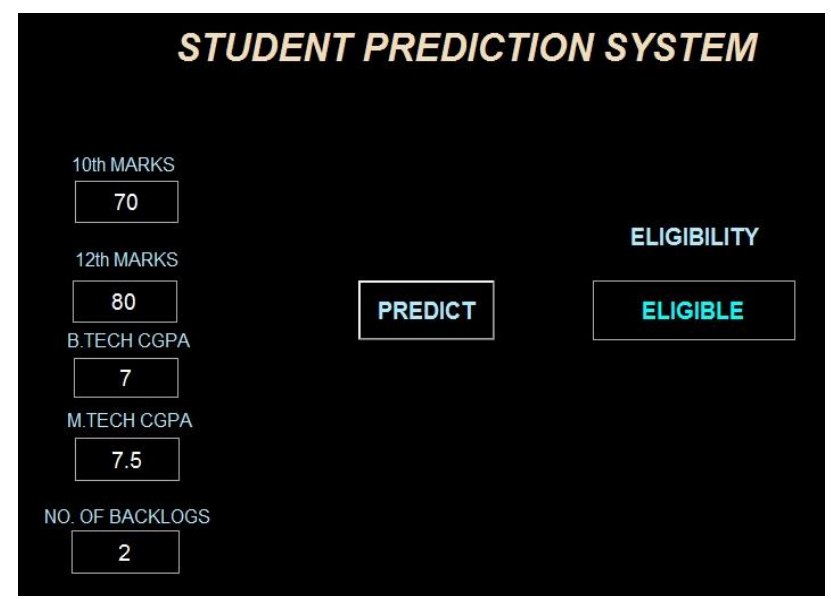

Fig.9. Output of Classified Individual student result on GUI.
In Fig.8, each input $\left(10^{\text {th }}\right.$ marks, $12^{\text {th }}$ marks, B.Tech CGPA, M.Tech CGPA, and number of backlogs) and output values which came by evaluating or classifying data set and finally it produces the output in the form of student is eligible for placement training or not it clearly showing in the last column and show a value for each student if value is greater than 2.1 than student is eligible for placement training otherwise he/she is not eligible for placement training and Fig.9 shows the GUI interface of student predicting system.

\section{CONCLUSION}

This system easily predicts and analyzes lot of student data set for predefined classes by using fuzzy logic which will definitely be a good asset to organization to analyze the information of huge number of student information and data sets. Future studies are required to investigate new hybrid models of fuzzy classification algorithms to improve the performance of prediction system.

\section{REFERENCES}

[1] Indriana Hidayah, Adhistya Erna Permanasari and Ning Ratwastuti, "Student Classification for Academic Performance Prediction using Neuro Fuzzy in a Conventional Classroom”, Proceedings of IEEE Conference Information Technology and Electrical Engineering, pp. 15, 2013.

[2] Satwanti Devi, Sanjay Kumar and Govind Singh Kushwaha, "An Adaptive Neuro Fuzzy Inference System for Prediction of Anxiety of Students", Proceedings of $8^{\text {th }}$ International Conference on Advanced Computational Intelligence, pp. 7 13, 2016.

[3] M. Alkhozae, "Students Performance Prediction System using Multi Agent Data Mining", International Journal of Data Mining and Knowledge Management Process, Vol. 4, No. 5, pp. 1-20, 2014.

[4] Q. Qiu and G. Sapiro, "Learning Transformations for Clustering and Classification", Journal of Machine Learning Research, Vol. 16, pp. 187-225, 2015.

[5] M. Sharma, "Data Mining : A Literature Survey", International Journal of Emerging Research in Management and Technology, Vol. 9359, No. 2, pp. 1-4, 2014.

[6] Mashael A. Al-Barrak and Muna Al-Razgan, "Predicting Students Final GPA Using Decision Trees: A Case Study", International Journal of Information and Education Technology, Vol. 6, No. 7, pp. 528-533, 2016.

[7] S.K. Yadav, "Data Mining : A Prediction for Performance Improvement of Engineering Students using Classification", World of Computer Science and Information Technology Journal, Vol. 2, No. 2, pp. 51-56, 2012.

[8] B. Minaei-bidgoli, D.A. Kashy, G. Kortemeyer and W.F. Punch, "Predicting Student Performance: An Application of Data Mining Methods with An Educational Web-Based System", Proceedings of $33^{\text {rd }}$ Annual Frontiers in Education, pp. 1-6, 2003.

[9] Bashir Khan, Malik Sikandar Hayat and Muhammad Daud Khattak, "Final Grade Prediction of Secondary School Student using Decision Tree", International Journal of Computer Applications, Vol. 115, No. 21, pp. 32-36, 2015. 